\title{
Assessing Attention Deficit by Binocular Rivalry
}

Juan Antonio Amador Campos ${ }^{1,5}$, Ph.D.

J. Antonio Aznar Casanova ${ }^{2,5}$, Ph.D.

Juan Jairo Ortíz Guerra ${ }^{3}$

Manuel Moreno Sánchez ${ }^{2}$, M.S.S.

Antonio Medina Peña ${ }^{4}$

${ }^{1}$ Department of Personality, Assessment and Psychological Treatments, Faculty of Psychology, University of Barcelona

${ }^{2}$ Department of Basic Psychology, Faculty of Psychology, University of Barcelona

${ }^{3}$ Psychiatry and Psychology Service, Sant Joan de Deu Hospital (Barcelona)

${ }^{4}$ Sant Domènec Savio Salesian School, Badalona (Barcelona)

${ }^{5}$ Institute for Brain, Cognition and Behavior (IR3C)

\section{Acknowledgements}

The study was funded by grant FAKO-2009 from the Alicia Koplowitz Foundation (Dr. Amador) and grant PSI2009-11062-PSIC from the Spanish Ministry of Science and Innovation (MICINN) (Dr. Aznar). 
Attention is a mechanism through which the brain exerts voluntary control over perceptual, cognitive and motor activity by activating or inhibiting other processes required to achieve goals (Posner, 1995). Attending to a stimulus requires the ability to select and separate it from other irrelevant stimuli, whether internal or external. This ability has been related to processes of inhibitory control (Luna, Garver, Urban, Lazar, \& Sweeney, 2004). Control processes may be automatic (reflex) or intentional (voluntary) (Marzi, 1999). In terms of human development, automatic processes are the first to be established and may serve as the basis for voluntary control (Aksan \& Kochanska, 2004).

Attention deficit hyperactivity disorder (ADHD) is a neurobiological disorder of childhood onset and it is common in children, adolescents and adults (American Psychiatric Association, APA, 2000; Polanczyk, De Lima, Horta, Biederman, \& Rohde, 2007). One of the most influential models of ADHD proposes that the central deficit of this disorder is the inability to inhibit responses (Barkley, 1997). The fourth edition revised of the Diagnostic and Statistical Manual of Mental Disorders (DSM-IV-TR, APA, 2000) distinguishes between three types of ADHD: Combined (ADHD-C), Predominantly Inattentive Type (ADHD-I) and Predominantly Hyperactive-Impulsive Type (ADHD-HI). Some researchers consider that ADHD-I and ADHD-C reflect distinct disorders rather than variants of a single disorder, since they differ in terms of the type of attentional processes that are altered and in the brain circuits associated with these alterations (Barkley, 1997; Diamond, 2005; Hynd, Lorys, Semrud-Clikeman, Nieves, Huettner, \& Lahey, 1991; Milich, Balentine, \& Lynam, 2001).

Over the last two decades a number of studies have examined inhibitory control in subjects with ADHD and controls, this being done by means of the stop paradigm in relation to motor tasks (Alderson, Rapport, Sarver, \& Kofler, 2008; Lijffijt, Kenemans, Verbaten, \& van Engeland, 2005; Oosterlaan, Logan, \& Sergeant, 1998) and by analyzing eye movements when the task requires visual attention. The most important findings of these studies are: (1) compared with 
controls, ADHD groups show considerable variability in their responses; (2) subjects with ADHD take longer to respond to stop stimuli in motor tasks than do people without the disorder; and (3) ADHD groups have greater difficulty with the voluntary control of eye movements, and find it more difficult to inhibit the direction of their gaze toward irrelevant or distractor stimuli (for a review, see Luna, Velanova, \& Geier, 2008; Rommelse, Van der Stigchel, \& Sergeant, 2008).

The diagnosis of attention disorders is controversial and the question of what constitutes an attention deficit has yet to be unequivocally answered. Hence it is important to define objective indicators that could help in reaching a diagnosis. There is experimental evidence that people with ADHD do not have problems with some automatic attentional processes (Huang-Pollock, \& Nigg, 2003). However, they do present difficulties with voluntary processes such as redirecting attention toward new stimuli, maintaining attention and task perseverance (Diamond, 2005).

When multiple stimuli compete to produce a representation in visual awareness our attention must select a sample of these stimuli for processing (Tong, 2001). Thus, when two dissimilar images are presented dichoptically, as occurs in binocular rivalry (BR) tasks, conscious awareness alternates, every few seconds, between one image and the other, such that one of the two competing images is suppressed. Does this correspond to a spontaneous or reflex (involuntary) inhibition of one of the visual channels? BR provides a genuine way of accessing visual awareness and selective attention (Alais \& Blake, 2005; Blake \& Logothetis, 2002; Paffen, Alais, \& Verstraten, 2006; Tong, 2001). As stimulus selection is a property that is shared by attention and BR it is assumed that the two are related (Tong, Meng, \& Blake, 2006). Various studies (Blake \& Logothetis, 2002; Meng \& Tong, 2004; Mitchell, Stoner, \& Reynolds, 2004; Ooi \& He, 2003) have analyzed the role played by attention in BR, and the results suggest that when a stimulus is attended to it is more likely: 1) that this stimulus becomes the dominant percept in awareness; and 2) that the duration of this perceptual dominance is prolonged, 
even though the permanence of the percept cannot be maintained for too long, since automatic alternations take place .

Measures of BR (number of alternations and the duration of exclusive dominance) may reveal certain types of adaptations that underlie a change in visual awareness. Meng and Tong (2004) examined the effects of attention on the duration of exclusive dominance periods and found that it affected the alternation rate but not the duration of dominance periods. There are a number of questions that follow from the above findings. Firstly, do children with ADHD differ from controls as regards the rate of perceptual alternations and the duration of exclusive dominance periods? Secondly, if ADHD children have greater difficulties with voluntary inhibitory control, what would happen when they fix their attention, in sustained fashion, on rival images? Thirdly, if the alternation rate and the duration of exclusive dominance periods are related to automatic or involuntary inhibition, what patterns of alternation/duration of exclusive dominance would we expect to find among children with ADHD and controls?

The present study aims to contribute to our understanding of how the inhibitory mechanism works in individuals with ADHD, providing evidence from the experimental BR paradigm. It is hypothesized that, in the context of BR, the frequency and duration of periods of suppression of a percept could be modulated by a process of inhibition that would act involuntarily. Were this the case, this inhibition could be interpreted as the mechanism responsible for the lower rate of alternations (longer dominance periods) when the observer is presented with a novel stimulus. For this hypothesis to be confirmed the results would need to show that repeated and prolonged exposure to the same stimulus leads to a decrease in the activity of this inhibitory mechanism, such that the number of alternations would increase as their duration became shorter. This pattern of results would be expected to be more widespread among ADHD individuals compared with controls. In order to test this hypothesis two experiments were conducted. 


\section{Experiment 1}

\section{Method}

\section{Participants}

The sample comprised 122 participants aged between 6 and 15 years (mean $=10.33$ years; SD $=2.74)$, each of whom was assigned to one of the following groups:

ADHD group. This was formed by 43 participants, 31 with ADHD-C (mean age $=10.27$ years, SD $=2.32 ; 26$ males) and 12 with ADHD-I (mean age $=11.29$ years, SD $=2.54 ; 10$ males), all of whom were recruited from the psychiatry and psychology service of a public hospital. The diagnosis was made in accordance with DSM-IV-TR (APA, 2000) criteria, by means of interview (Clinical Interview-Parent Report Form; Barkley \& Murphy, 2006) and on the basis of scores on both an ADHD questionnaire (Amador, Forns, Guàrdia, \& Peró, 2006) and the Conners' Rating Scales-Revised (Conners, 1997), which were completed by the participants' parents and teachers. The classification of the type of ADHD was established using the results of the interview and the questionnaire/scale scores. Participants were classified as ADHD-I if they met the criteria for inattention, but not those for hyperactivity-impulsivity, in the interview and according to the scales completed by parents and teachers (i.e., six or more symptoms of inattention and fewer than six symptoms of hyperactivity-impulsivity, scored as 2 or 3 on the ADHD questionnaire for parents and teachers; T scores $>65$ on the Cognitive problems/Inattention scale and the ADHD Index, and T scores $<65$ on the Hyperactivity scale of the Conners' scales for parents and teachers). Participants with ADHD-C met the criteria for both inattention and hyperactivityimpulsivity (six or more symptoms of inattention and of hyperactivity-impulsivity, scored as 2 or 3 on the ADHD questionnaire for parents and teachers; $T$ scores $>65$ on the Cognitive problems/Inattention scale, the Hyperactivity scale and the ADHD Index of the Conners' scales for parents and teachers).

Control group. This comprised 79 participants (mean age $=10.23$ years, SD $=2.92 ; 33$ males). They all presented fewer than six symptoms of inattention and hyperactivity-impulsivity, as 
assessed by their parents and teachers using the ADHD questionnaire, and they also had T scores below 60 on the Cognitive problems/Inattention scale, the Hyperactivity scale and the ADHD Index of the Conners' scales for parents and teachers.

The ADHD-C, ADHD-I and control groups were equivalent in terms of age $[F(2,121)=.880$, $\mathrm{p}=.42$; a posteriori contrasts (Tukey's HSD) showed no significant differences between the three groups]. For all participants, visual acuity (Snellen chart) was either normal or corrected to normal (with contact lenses or glasses), and they all had normal stereoscopic acuity (Titmus test). The exclusion criteria for both groups were: Total IQ $\leq 80$ according to the WISC-IV (Wechsler, 2003), tic disorders, neurological disorders, pervasive developmental disorders, oppositional defiant disorder, conduct disorder, anxiety and mood disorders, learning disorders, or speech and language disorders. Participation was voluntary in all cases and an informed consent form was signed by the children's parents or legal guardians. The principles of the 1975 Helsinki Declaration (revised in Tokyo in 2004) were adhered to throughout the study.

\section{Measures}

Clinical Interview-Parent Report Form (Barkley \& Murphy, 2006). This was used to collect information from the parents of children or adolescents with ADHD in the clinical sample. The interview covers seven areas: family composition, parental concerns about the child, review of DSM-IV-TR childhood disorders, parent management methods, school history, child's psychological and social strengths, and family history of psychiatric and learning problems. ADHD Questionnaire (Amador et al., 2006). This comprises 18 items that assess the symptoms of ADHD as listed in DSM-IV. Parents and teachers are asked to rate the frequency and intensity of symptoms on a scale from 0 to $3(0=$ Not true at all; $3=$ Very much true, this happens to him/her almost all the time). For both sets of ratings (parent and teacher) the 18 symptoms of ADHD are grouped into two factors: Inattention and Hyperactivity-impulsivity 
(Amador-Campos, Forns-Santacana, Martorell-Balanzó, Guàrdia-Olmos, \& Peró-Cebollero, 2005). The questionnaire shows high reliability, with values being slightly higher for teachers (Cronbach's alpha between .948 and .957) than for parents (Cronbach's alpha between .858 and .892).

Conners' Rating Scales-Revised (Conners, 1997). These scales assess symptoms and behaviors associated with ADHD. There are forms for parents and teachers, covering an age range for subjects of 3 to 17 years. The presence and severity of each behavior is rated on a scale from 0 to $3(0=$ Not true at all, never, seldom; $3=$ Very much true, very frequent $)$. The factor analyses of the short forms, which are those used in the present study, reveal four factors for parents and teachers: Oppositional, Cognitive problems/Inattention, Hyperactivity and the ADHD Index. The internal consistency of the different scales ranges between moderate and excellent for both the parent report (.86 to .94$)$ and the teachers' version (.88 to .95 ). Test-retest reliability over a 6-8 week interval ranges between moderate and high (parent report: .62 to .85 ; teacher report: .72 to .92$)$.

Wechsler Intelligence Scale for Children, fourth edition (WISC-IV; Wechsler, 2003). Eight of the ten main tests were applied, the exceptions being Comprehension and Concepts. The indices for working memory (WM), processing speed (PS) and pro rata full-scale intelligence quotient (PFIQ) were therefore calculated on the basis of the eight tests applied. This combination provides an adequate estimate of full-scale IQ (Glass, Ryan, Bartels, \& Morris, 2008).

Binocular rivalry stimulus and task

The stimulus used in the binocular rivalry task (BRT; Aznar-Casanova, 2010) was a bistable image comprising two superimposed layers. One, which was oriented horizontally, contained a sinusoidal variation of luminance in the red spectral band, with a spatial frequency of four cycles. The other, oriented vertically, had a sinusoidal variation of luminance in the green band, with a spatial frequency of five cycles (see Figure 1). These two overlapping images formed an anaglyph, which when presented dichoptically produced the phenomenon of 
binocular rivalry. The stimulus was viewed from a distance of $60 \mathrm{~cm}$, such that it subtended a visual angle of $4^{\circ}$ on the observer's retina.

\section{INSERT FIGURE 1 ABOUT HERE}

\section{Apparatus}

The BRT was applied using a Lenovo ThinkPad T510 PC. The stimuli were presented on a 22inch screen, with a spatial resolution of $1024 \times 768$ pixels. Eye movements were controlled by an eye tracker (iViewRED-120 Hz, SensoMotoric Instruments GMBH, Teltow, Germany). Redgreen filter glasses were used to present the images dichoptically. The participants' responses were recorded by means of a response box, and specific software (Experiment CenterTM, v. $2.5^{\odot}$, by $\mathrm{SMI}$ ) was used to control the time of stimuli presentation and the fixation point, and also to record the number of alternations in dominance/suppression and the duration of exclusive dominance periods.

\section{Procedure}

Participants were assessed in a room, either of the hospital or their school, which provided similar environmental conditions. They were seated $60 \mathrm{~cm}$ from the screen, at the center of which was presented the stimulus designed for the BRT. They were instructed to look continuously at the center of the image for $1 \mathrm{~min}$, without moving their head or eyes, or blinking. These factors were controlled through the use of a chin-rest and an eye tracker. The specific procedure was as follows. After the required calibration the apparatus presented a white image with a cross drawn at the center, at which the participant had to look for $2 \mathrm{~s}$; the stimulus, comprising the two superimposed rival images (Figure 1), was then immediately presented. The participant was asked to press the button that was identified with vertical lines 
on the response box as soon as he/she exclusively perceived a vertical grating (red-green) and to keep it pressed as long as he/she continued to see this image. Similarly, subjects were asked to press the corresponding button for the horizontal grating (yellow-green), and to keep it pressed as long as he/she continued to see this image. Subjects were asked to only press a button when they perceive a clear dominant image, but not when piecemeal rivalry was perceived. The participants received some previous training in the two response options with vertical and horizontal arrows as stimuli. For each trial we recorded the rate of perceptual alternations and the duration of exclusive dominance periods. Only those trials with calibrations $\leq 1^{\circ}$ in the vertical and horizontal axes were analyzed. Blink times were excluded from the analysis.

For each trial we recorded the rate of perceptual alternations and the duration of exclusive dominance periods. Only those trials with calibrations $\leq 1^{\circ}$ in the vertical and horizontal axes were analyzed. Blink times were excluded from the analysis. The recordings with very low time percentages, $\leq 10 \%$, were also excluded from the analysis. In each group, the total time percentages recorded and analyzed for the whole BRT were as follows: $A D H D-C=87.84 \%$, ADHD-I $=91.01 \%$ and Control $=95.43 \%$. No significant differences were found between the time percentages/OK? of the ADHD-C and Control groups $(z=-1.106, p<.1446)$, between ADHDI and Controls ( $z=-0.460, \mathrm{p}<.3228)$, or between ADHD-C and ADHD-I $(z=0.297, \mathrm{p}<.3859)$.

Figure 2 shows the sequence of a trial for this task. The BRT was applied at two points in time (initial and final) which were separated by another task, the one applied in Experiment 2. 


\section{Data analysis}

We conducted a 3 (groups) $\times 2$ (time points) mixed factor ANOVA, taking the variable 'group' as the between-subjects factor and the variable 'time point' as the within-subjects (repeated measures) factor, and controlling for the influence of age as a covariate. The dependent variables were the mean number of alternations and the mean duration in milliseconds (ms) of exclusive dominance periods.

\section{Results}

As regards the alternation rate the ANOVA revealed significant differences for the factors 'time point' $[F(1,118)=11.594 ; p<.001]$, 'group' $[F(2,118)=7.693 ; p<.001]$ and 'age' $[F(1,118)=18.026$; $p<.001]$. The interaction effects of 'time point $x$ group' $[F(2,118)=3.215 ; p<.04]$ (Figure 3 ) and 'time point $x$ age' $[F(1,118)=8.112 ; p<.005]$ were also significant. A posteriori contrasts (Tukey's test) were also performed at time point 1 (initial) and time point 2 (final). The comparison of means for time point 1 (Figure 3) showed significant differences [t(118)=-6.49, SE=1.275; $p<.001$ ] between the ADHD-C group $(M=13.468 ; S E=1.056)$ and controls $(M=19.992 ; S E=0.661)$, but not between the ADHD-I group ( $M=17.12 ; \mathrm{SE}=1.707)$ and controls. At time point 2 there were no significant differences between any of the three groups: the alternation rate among participants with ADHD (ADHD-C and ADHD-I groups) was statistically equivalent to that of controls.

\section{INSERT FIGURE 3 ABOUT HERE}

When using the mean duration time of exclusive dominance periods as the dependent variable the ANOVA showed that of the main effects only 'age' reached statistical significance $[F(1,118)=40.945 ; p<.001]$. As regards the interaction effects, only 'time point $x$ age' was significant $[F(1,118)=5.339 ; p<.023]$. 


\section{Experiment 2}

The BR paradigm differs from other experimental paradigms (e.g., visual search, go-no-go, stop-signal task, etc.) in that attention is fixed, in a sustained fashion, on the center of rival images which alternate with different rates and periodicity for each subject, and generally without distractor stimuli being presented. In order to verify the robustness of alternation rate as an indicator of $\mathrm{BR}$, and to analyze in greater depth the duration of exclusive dominance periods, we designed Experiment 2. Here, two variations were introduced into the BRT: (1) a dynamic exogenous distractor was added; and (2) the number of task trials was increased, thereby enabling us to test the sustained attention of participants. By studying the effects of these new factors on the behavioral measures of the BRT (number of alternations and the duration of exclusive dominance periods) we hope to increase our understanding of the inhibitory mechanism in BR.

\section{Method}

\section{Participants}

Participants were the same 122 individuals who took part in Experiment 1.

\section{Stimulus, task and apparatus}

The apparatus and stimulus were the same as described in Experiment 1. However, the BRT was modified slightly, adding a small black circle (5 $\mathrm{mm}$ in diameter) which appeared regularly around the stimulus, thereby acting as a distractor (Figure 1). In this way a new task was designed (Aznar-Casanova, 2010) to evaluate the observer's susceptibility to distraction (caused by an exogenous stimulus) and the consequences of prolonging the task. The task comprised eight trials in which the stimulus described in Experiment 1 was presented along with the distractor, whose position changed temporally every $3 \mathrm{~s}$.

\section{Procedure}


The new BRT involved eight trials, each lasting $24 \mathrm{~s}$ and separated by a rest period of $10 \mathrm{~s}$. Each trial began with the fixation point being presented for $2 \mathrm{~s}$ (see Figure 2). The number of alternations, the duration of exclusive dominance periods, and the number of fixations and blinks were recorded in all trials. The whole task lasted a total of 5 min. As in Experiment 1, only those trials with calibrations $\leq 1^{\circ}$ in the vertical and horizontal axes were analyzed. Blink times were excluded from the analysis.

\title{
Data analysis
}

We conducted a 3 (groups) $\times 8$ (trials) between-within ANOVA for repeated measures, taking the variable 'group' as the between-subjects factor and the variable 'trial' as the withinsubjects (repeated measures) factor, and controlling for the influence of age as a covariate. The dependent variables were the alternation rate and the mean duration time of exclusive dominance periods.

\section{Results}

As regards the alternation rate the ANOVA revealed significant differences for the factors 'trial' $[F(7,826)=4.485 ; p<.001]$, 'group' $[F(2,118)=10.145 ; p<.001]$ and 'age' $[F(1,118)=21.438$; $p<.001]$. The interactions 'group $x$ trial' $[F(14,826)=2.770 ; p<.001]$ (Figure 4) and 'trial $x$ age' $[F(7,826)=2.204 ; p<.04]$ were also significant.

In order to examine the 'group $\mathrm{x}$ trial' interaction in greater depth we analyzed the data trends over time. This revealed that the best fit to the data was provided by a logarithmic model. Table 1 shows the equations of the functions that best fitted the data, as well as the goodness of fit index, expressed in terms of the coefficient of determination $\left(R^{2}\right)$.

\author{
INSERT TABLE 1 ABOUT HERE
}

INSERT FIGURE 4 ABOUT HERE 
Participants in the ADHD-C group differed from those in the control group, with the ADHD-I group being intermediate between the two. In all cases the trend was towards a greater number of alternations as the task duration increased (i.e., greater number of trials). However, the range of variation for the ADHD-C group $(6.0-8.0)$ was wider than that for controls $(8.2-$ 9.5), while that of the ADHD-I group $(7.8-8.6)$ fell between, and overlapped, the two. In addition, the rate of change, expressed by the $b$ coefficient of the equation, was greater for the ADHD-C group (1.3076) than for the ADHD-I group (0.5721), with the latter value being similar to that obtained by controls (0.5519). The value of $R^{2}$ expresses the proportion of variance (in the number of alternations) that is explained by task prolongation (greater number of trials), and the results showed that this value was higher in the ADHD-C group (0.8146) than in both the ADHD-I (0.5121) and control (0.6091) groups.

The same between-within ANOVA for repeated measures was then applied with the mean duration time of exclusive dominance periods as the dependent variable. As regards the main effects this ANOVA showed that participants differed significantly in relation to 'age' $[F(1,118)=46.044 ; p<.001]$. There was also a significant 'group $x$ trial' interaction effect $[F(14,826)=4.924 ; p<.001]$. Figure 5 shows, for each group, how the periods of exclusive dominance changed over time.

INSERT FIGURE 5 ABOUT HERE

On the first trial the mean duration of exclusive dominance was significantly higher for the clinical groups (ADHD-C = $2966 \mathrm{~ms} ;$ ADHD-I = $2754 \mathrm{~ms}$ ) than for the control group (2407 ms). However, as the task duration increased, the duration of dominance periods tended to decrease logarithmically in the clinical groups, while remaining relatively stable in the control group. 


\section{Discussion}

In order to test the hypothesis that the rate of change between periods of suppression of a percept in a situation of binocular rivalry might be modulated by a process of involuntary inhibition, this study recorded two standard measures of BR: the rate of perceptual alternations and the duration of exclusive dominance of the percepts. How might we interpret the fact that an individual produces more or fewer alternations in his or her visual awareness? What might it mean that one of the rival images is retained for longer or for less time in a person's conscious experience? It has been suggested that perceptual alternation may involve a shift in attention (Blake \& Logothetis, 2002; Meng \& Tong, 2004); in other words, the individual's attention must choose between two possible percepts and/or there needs to be a reflex (or involuntary) inhibition of one of them.

With respect to perceptual alternations the results of Experiment 1 showed that repetition of the BRT had a differential effect on the groups. Specifically, the number of alternations was significantly lower in the ADHD-C group compared with controls. In other words, the processes of automatic inhibition, represented by perceptual alternation in the BRT, occur more often among control and ADHD-I children than they do among those with ADHD-C. However, this is only the case at the start of the BRT (time point 1), and as the number of trials increases the number of alternations produced by the ADHD-C group also increases progressively, the rate of change being twice that observed among the ADHD-I and control groups (which were statistically equivalent). The results also showed that the number of trials explained $81.5 \%$ of the variability in the number of alternations in the ADHD-C group, whereas in the ADHD-I and control groups it explained $51.2 \%$ and $60.1 \%$, respectively. In summary, prolongation of the BRT led to greater automatic inhibition of the percepts and had a significant effect on the alternation rate, especially in the ADHD-C group.

There are three possible explanations for these results. The first is that the differences in the rate of alternations may be due to differences in the information processing speed (PS). The 
control group obtained higher scores than the clinical groups (ADHD-C and ADHD-I) on the Processing Speed (PS) index of the WISC-IV (Wechsler, 2003). The PS index combines scores of two tests, Digit Symbol-Coding and Symbol Search, which both use visual stimuli, and measures the ability to process visual information quickly and efficiently [ADHD-C: $M=98.74 ; S D=10.50$; ADHD-I: $M=100.17 ; 74, S D=8.95 ;$ Control: $M=112.51 ; 74, S D=13.38 ; F(2.119)=16.351 ; p<$ $\left..001 ; \eta^{2}=0.216\right]$. The participants who processed the information more slowly were the ones who obtained lower alternation rates. However, if the alternation rate depended only on the processing speed, the rate of the ADHD-I group would be equivalent to that of the ADHD-C group and not to that of the control group, as occurs in this case. Probably, in addition to information processing speed, the capacity to inhibit a percept is in operation and influences the perceptual alternation. Our results are in accordance with those of Fillmore, Milich and Lorch (2009) who studied the processes of voluntary and automatic inhibition using a voluntary saccade suppression task and an inhibition of return task (IOR), and found that children with ADHD presented longer reaction times on both tasks than the control group. Though there were no significant differences between ADHD-C and ADHD-I groups in the saccade suppression task, significant differences were found between the ADHD-C and ADHD-I groups on the IOR task, that is, the one that involved an automatic inhibition process. The ADHD-C group presented more difficulties on the IOR task than the ADHD-I group. The ADHD-I group displayed impaired reflexive inhibition, although the impairment was slightly lower than in the ADHD-C group. That is, the ADHD subtypes did not present differences in processes of voluntary inhibition control, but did present differences in automatic control. The second explanation is that repetition or familiarity with the stimulus facilitates stimulus processing, favors perceptual change, and increases the rate at which the percepts are automatically inhibited, especially among children with ADHD-C; that is, task training may subserve the processing speed and the automatic inhibition of percepts. The third possibility is that the presence of the distractor (the black circle) around the stimulus may favor perceptual 
alternation. Several studies (e.g., Chong \& Blake, 2006; Mitchell, Stone, \& Reynolds, 2004) have found that highlighting certain parts of a stimulus in a BRT favors the onset of binocular rivalry and alternation between percepts. It is possible that the appearance of an external stimulus had a particular effect on the participants with ADHD-C, the ones who showed the greatest rate of change. This may corroborate previous research showing that individuals with ADHD-C are more sensitive to the influence of exogenous distractors (Barkley, 1989, 1997; Luna et al., 2008, Rommelse et al., 2008). Therefore, it is possible that in the case of the ADHDC group the increase in the rate of alternations is a consequence both of the facilitatory effect of task repetition and of the appearance of the distractor.

As regards the duration of exclusive dominance periods the results of Experiment 1 revealed no significant differences between the groups. However, Experiment 2 showed there to be a significant 'group $\mathrm{x}$ trial' interaction effect. Thus, on the first trial the mean duration of exclusive dominance was significantly higher for the clinical groups (ADHD-C and ADHD-I) than for the control group. However, as the number of trials increased, the duration of dominance periods decreased logarithmically in the clinical groups, while remaining relatively stable over time in the control group. These results are consistent with those reported by Meng and Tong (2004), who also found that attention affected the alternation rate but not the duration of dominance periods. However, the significant 'group $\mathrm{x}$ trial' interaction effect observed in the present study indicates that prolongation of the BRT brings to light differences that can be attributed to the duration of dominance. These differences may be due to fluctuations in the processing speed and in the activity of the inhibitory mechanism over time. In the present study the greatest rate of fluctuation across the BRT was shown by participants with ADHD, especially those diagnosed as ADHD-C, who are the ones with the lowest PS index. These results are in line with previous findings derived from both motor inhibition tasks (Adams, Derefinko, Milich, \& Fillmore, 2008; Diamond, 2005; Fillmore et al, 2009; Lijffijt, et al., 2005; Nichols \& Waschbusch, 2004; Senderecka, Grabowska, Szewczyk, Gerc, \& Chmylak, 2012) and 
eye movement registers (Fillmore et al., 2009; Huang-Pollock, \& Nigg, 2003; Luna et al., 2008, Rommelse et al., 2008).

Finally, it should be noted that there are at least two important differences between the BRT described here and other tasks that have been used to investigate the inhibitory mechanism among individuals with ADHD: 1) in the BRT one needs to think in terms of involuntary (or covert) attention, rather than attention that is openly directed towards one or other of the images that are competing to enter visual awareness (Tong et al.,2006); and 2) in the BRT, switching between the two rival percepts seems to be more of an automatic (involuntary) process rather than one based on voluntary selection (van Ee, van Dam, \& Brouwer, 2005). In this regard, the differences observed between ADHD participants and controls may be related to the processing speed and a slower functioning of automatic inhibition, which may be responsible for the smaller number of alternations and the longer duration of exclusive dominance periods. Thus, this inhibition would be weakest in the ADHD-C group, followed by ADHD-I participants and then controls.

In summary, the BRT would seem to be a valuable experimental paradigm for demonstrating that the suppression of representations is an effect of automatic inhibition, since each of the images that are competing to enter awareness captures the observer's attention automatically. Herein lies the importance of the present data.

\section{References}

Adams, Z. W., Derefinko, K. J., Milich, R., \& Fillmore, M. T. (2008). Inhibitory functioning across ADHD subtypes: Recent findings, clinical implications, and future directions. Developmental Disabilities Research Reviews, 14, 268-275. 
Aksan, N., \& Kochanska, G. (2004). Links between systems of inhibition from infancy to preschool years. Child Development, 75, 1477-1490.

Alais, D., \& Blake, R. (2005). Binocular rivalry. Cambridge, MA: MIT Press.

Alderson, R. M., Rapport, M .D., Sarver, D. E., \& Kofler, M. J. (2008). ADHD and Behavioral Inhibition: A Re-examination of the Stop-signal Task. Journal of Abnormal Child Psychology, 36, 989-998.

Amador, J. A., Forns, M., Guàrdia, J., \& Peró, M. (2006). Estructura factorial y datos descriptivos del perfil de atención y del cuestionario TDAH para niños en edad escolar [Factor structure and descriptive data of Attention profile and ADHD questionnaire for schoolage children] Psicothema, 18, 696-703.

Amador-Campos, J.A., Forns-Santacana, M., Martorell-Balanzó, B., Guàrdia-Olmos, J., \& PeróCebollero, M. (2005). Confirmatory factor analysis of parents' and teachers' ratings of DSM-IV symptoms of attention deficit hyperactivity disorder in a Spanish sample. Psychological Reports, 97, 847-860.

American Psychiatric Association, APA, (2000). Diagnostic and Statistical Manual of Mental Disorders (text revision). Washington, DC: Author

Aznar Casanova, J. A. (2010). Test for the assessment of the attention-deficit by perceptual rivalry. Register of intellectual property nr. B-1055-10 (Spain).

Barkley, R. A. ( 1989). The problem of stimulus control and rule-governed behavior in children with attention deficit disorder with hyperactivity. In L. M. Bloomingdale \& J. M. Swanson (Eds.), Attention deficit disorders (Vol. 4, pp. 203-234). New York: Pergamon Press.

Barkley, R.A. (1997). ADHD and the nature of self-control. New York: Guilford Press. 
Barkley, R. A., \& Murphy, K. R. (2006). Attention-deficit hyperactivity disorders. A clinical workbook (3rd Ed.). New York: Guilford Press.

Blake, R., \& Logothetis, N. K. (2002). Visual competition. Nature Reviews Neuroscience, 3, 1321.

Chong, S. Ch. \& Blake, R. (2006). Exogenous attention and endogenous attention influence initial dominance in binocular rivalry. Vision Research, 46, 1794-1803.

Conners, C. K. (1997). Conners' Rating Scales-Revised. Toronto: Multi-Health Systems

Diamond, A. (2005). Attention-deficit disorder (attention deficit/ hyperactivity disorder without hyperactivity): A neurobiologically and behaviorally distinct disorder from attention-deficit/hyperactivity disorder (with hyperactivity). Development and Psychopathology, 17, 807-825.

Fillmore, M. T., Milich, R., \& Lorch, E. P. (2009). Inhibitory deficits in children with attention deficit/hyperactivity disorder: Intentional versus automatic mechanisms of attention. Development and Psychopathology 21, 539-554.

Glass, L. A., Ryan, J. J., Bartels, J. M., \& Morris, J. (2008). Estimating WISC-IV indexes: Proration versus linear scaling. Journal of Clinical Psychology, 64, 1175-1180.

Huang-Pollock, C. L., \& Nigg, J. T. (2003). Searching for the attention deficit in attention deficit hyperactivity disorder: The case of visuospatial orienting. Clinical Psychology Review, $23,801-830$.

Hynd, G., Lorys, W., Semrud-Clikeman, M., Nieves, N., Huettner, M., \& Lahey, B. (1991). Attention deficit disorder without hyperactivity: A distinct behavioral and neurocognitive syndrome. Journal of Child Neurology, 6, 37-43. 
Lijffijt, M., Kenemans, J..L., Verbaten, M. N., \& van Engeland, H. (2005). A meta-analytic review of stopping performance in attention-deficit/hyperactivity disorder: Deficient inhibitory motor control? Journal of Abnormal Child Psychology, 114, 216-222.

Luna, B., Garver, K. E., Urban, T. A., Lazar, N. A., \& Sweeney, J. A. (2004). Maturation of cognitive processes from late childhood to adulthood. Child Development, 75, $1357-$ 1372.

Luna, B., Velanova, K., \& Geier, C. F. (2008). Development and eye-movement control. Brain and Cognition, 68, 293-308.

Marzi, C. A. (1999). Neuropsychology of attention. In G. Denes \& P. Luigi (Eds.), Handbook of clinical and experimental neuropsychology (pp. 509-524). Hove, UK: Psychology Press/Erlbaum/Taylor \& Francis.

Meng, M., \& Tong, F. (2004). Can attention selectively bias bistable perception? Differences between binocular rivalry and ambiguous figures. Journal of Vision, 4, 539-551.

Milich, R., Balentine, A. C., \& Lynam, D. R. (2001). ADHD combined type and ADHD predominantly inattentive type are distinct and unrelated disorders. Clinical Psychology: Science and Practice, 8, 463-488.

Mitchell, J. F., Stoner, G. R., \& Reynolds, J. H. (2004). Object-based attention determines dominance in binocular rivalry. Nature, 429, 410-413.

Nichols, S. L., \& Waschbusch, D. A. (2004). A review of the validity of laboratory cognitive tasks used to assess symptoms of ADHD. Child Psychiatry \& Human Development, 34, 297315.

Ooi, T. L. \& He, Z. J. A. (2003). Distributed intercortical processing of binocular rivalry: psychophysical evidence. Perception, 32, 155-166. 
Oosterlaan, J., Logan, G. D., \& Sergeant, J.A. (1998). Response inhibition in AD/HD, CD, comorbid $A D / H D+C D$, anxious and control children: A meta-analysis of studies with the stop task. Journal of Child Psychology and Psychiatry, 39, 411-425.

Paffen, C. L. E., Alais, D. , \& Verstraten, F. A. J. (2006). Attention speeds binocular rivalry. Psychological Science, 17, 752-7566.

Polanczyk, G., De Lima, M. S., Horta, B. L., Biederman, J., \& Rohde, L.A. (2007). The worldwide prevalence of ADHD: a systematic review and metaregression analysis. American Journal of Psychiatry, 164, 942-948.

Posner, M. (1995). Attention in cognitive neuroscience: An overview. In Gazaniga M. S. (Ed.), The cognitive neuroscience (pp. 615-624). Cambridge: MIT Press.

Rommelse, N. N. J., Van der Stigchel, S., \& Sergeant, J. A. (2008). A review on eye movement studies in childhood and adolescent psychiatry. Brain and Cognition, 68, 391-414.

Senderecka, M., Grabowska, A., Szewczyk, J., Gerc, K., \& Chmylak, R. (2012). Response inhibition of children with ADHD in the stop-signal task: An event-related potential study. International Journal of Psychophysiology, 85, 93-105.

Tong, F. (2001). Competing Theories of Binocular Rivalry: A Possible Resolution. Brain and Mind, 2, 55-83.

Tong, F., Meng, M., \& Blake, R. (2006). Neural bases of binocular rivalry. TRENDS in Cognitive Sciences, 10, 502-511.

van Ee, R., van Dam, L. C. J., \& Brouwer, G. J. (2005). Voluntary control and the dynamics of perceptual bistability. Vision Research 2005, 45, 41-55. 
Wechsler, D. (2003). WISC-IV. Wechsler Intelligence Scale for Children (4th ed.). San Antonio,

TX: Harcourt Assessment. 
Table 1. Equations of the functions that best fitted the data for each group and their corresponding coefficients of determination $\left(R^{2}\right)$.

\begin{tabular}{|l|l|l|}
\hline \multicolumn{1}{|c|}{ Group } & \multicolumn{1}{|c|}{ Equation } & \multicolumn{1}{c|}{$\mathrm{R}^{2}$} \\
\hline ADHD-C & $\mathrm{y}=1.3076 \ln (\mathrm{x})+5.6942$ & $\mathrm{R}^{2}=0.8146$ \\
\hline ADHD-I & $\mathrm{y}=0.5721 \ln (\mathrm{x})+7.795$ & $\mathrm{R}^{2}=0.5121$ \\
\hline Control & $\mathrm{y}=0.5519 \ln (\mathrm{x})+8.6139$ & $\mathrm{R}^{2}=0.6091$ \\
\hline
\end{tabular}

ADHD-C = Attention Deficit Hyperactivity Disorder Combined Type; ADHD-I = Attention

Deficit Hyperactivity Disorder Predominantly Inattentive Type. 


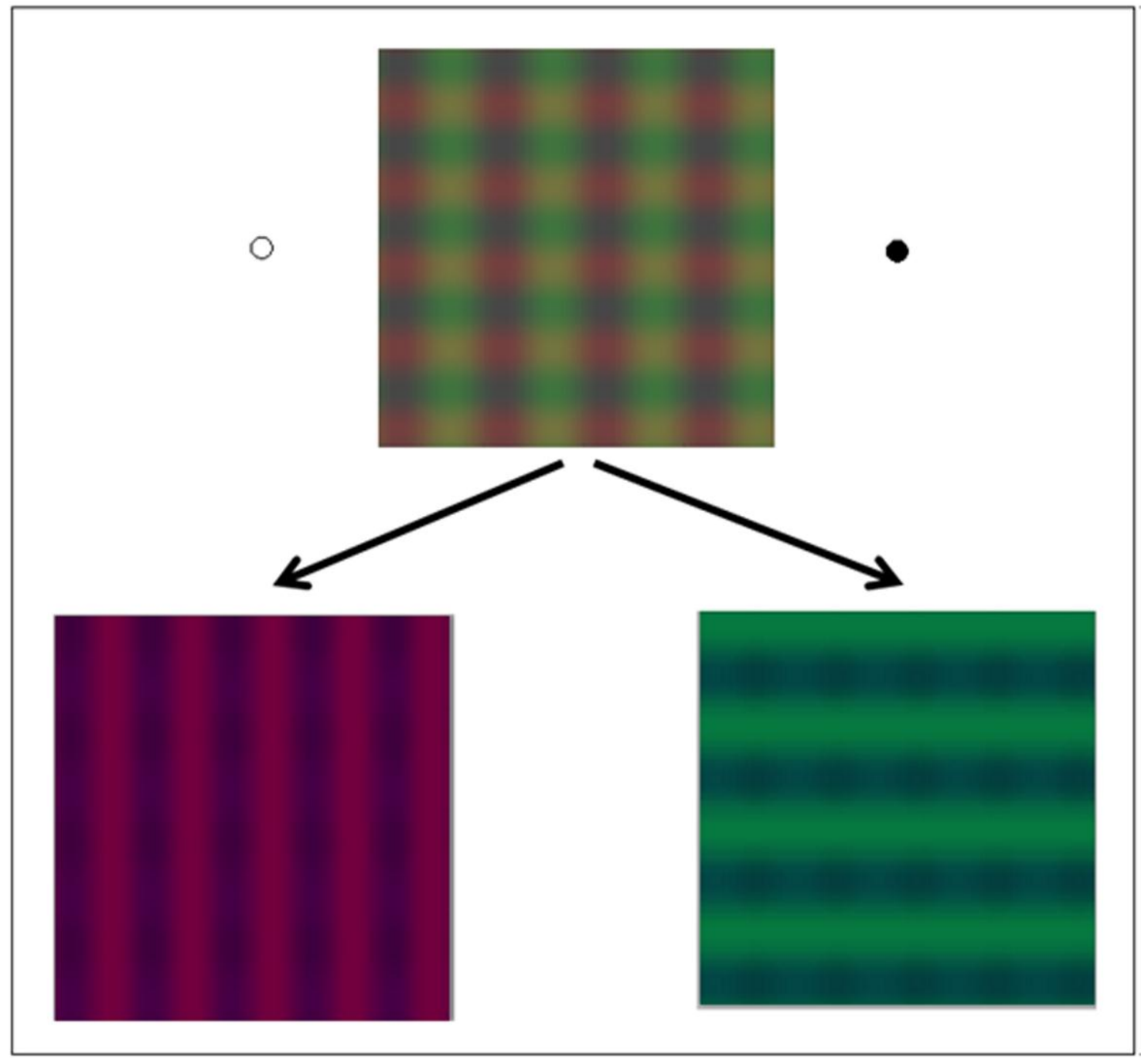

Figure 1. Bi-stable image used as the stimulus in the BRT of Experiment 1. It comprises two layers (upper part) that form a compound image: a grating with a sinusoidal variation of luminance $(4 \mathrm{c} / \mathrm{deg})$ in the red spectral band (bottom left) and a grating with a sinusoidal variation of luminance $(5 \mathrm{c} / \mathrm{deg})$ in the green band (bottom right). The black circles (filled and empty) indicate the positions of the periodically alternating exogenous distractor (left-right or up-down) that was introduced in Experiment 2. 


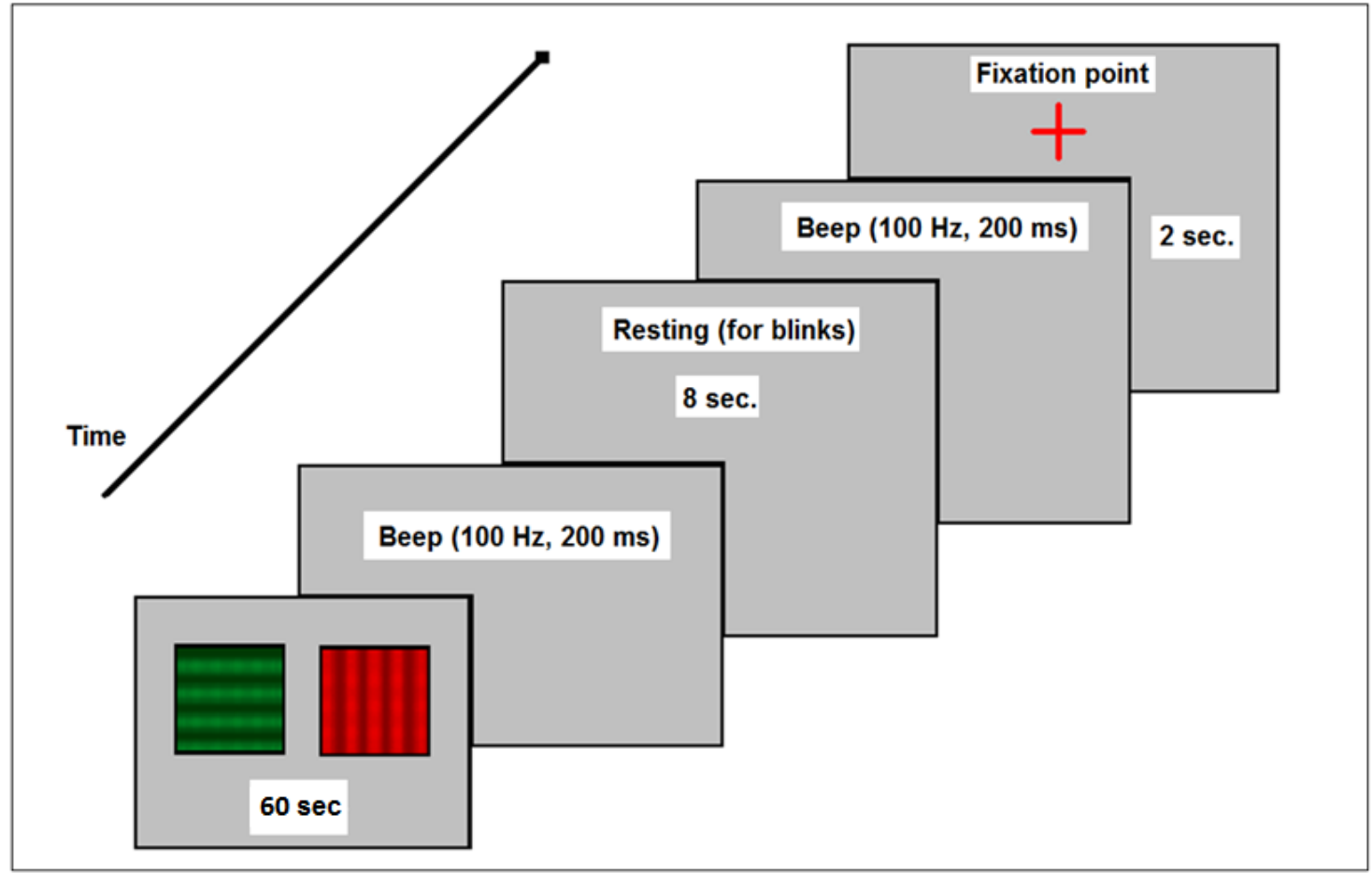

Figure 2. Sequence of a trial conducted under the BR paradigm. The figure shows the presentation of stimuli during the task and the brief rest periods that allow blinking. 


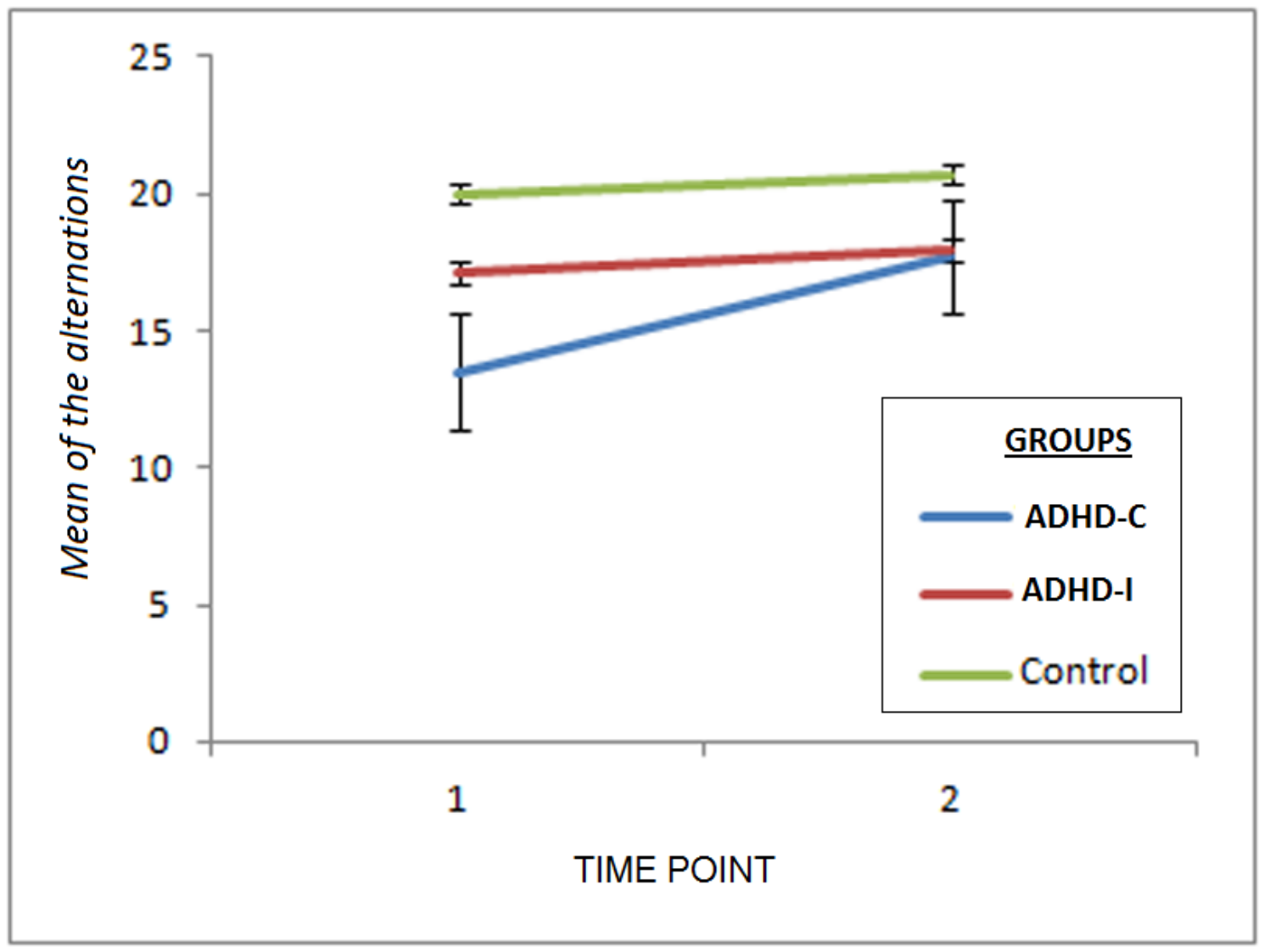

Figure 3. 'Group $x$ time point' interaction effect on the rate of alternations (dominance/suppression). The bars show the standard error. 


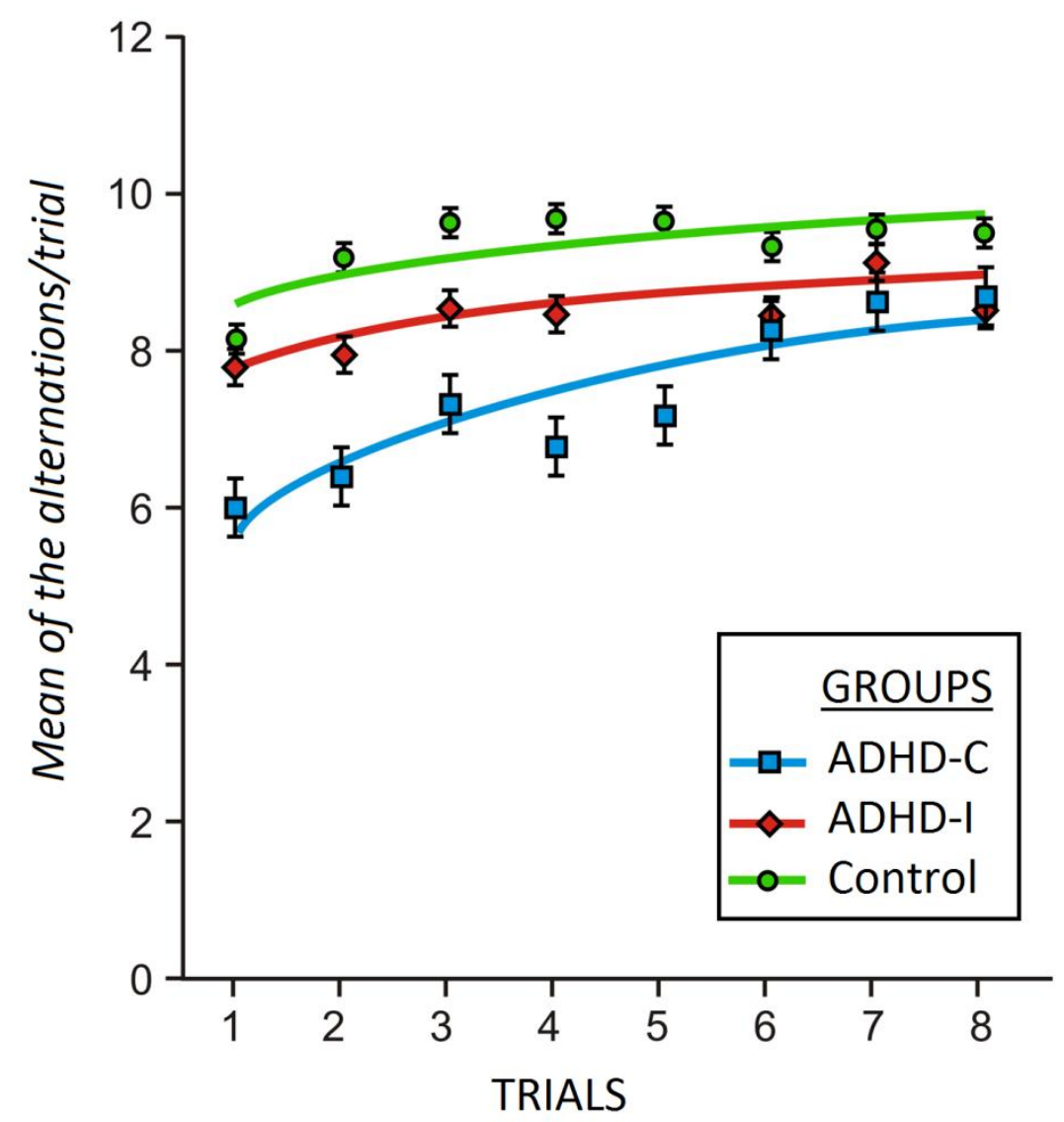

Figure 4. Representation of the shift in attention over time, indicated by the change in alternation rate according to the number of trials. The bars show the standard error. 


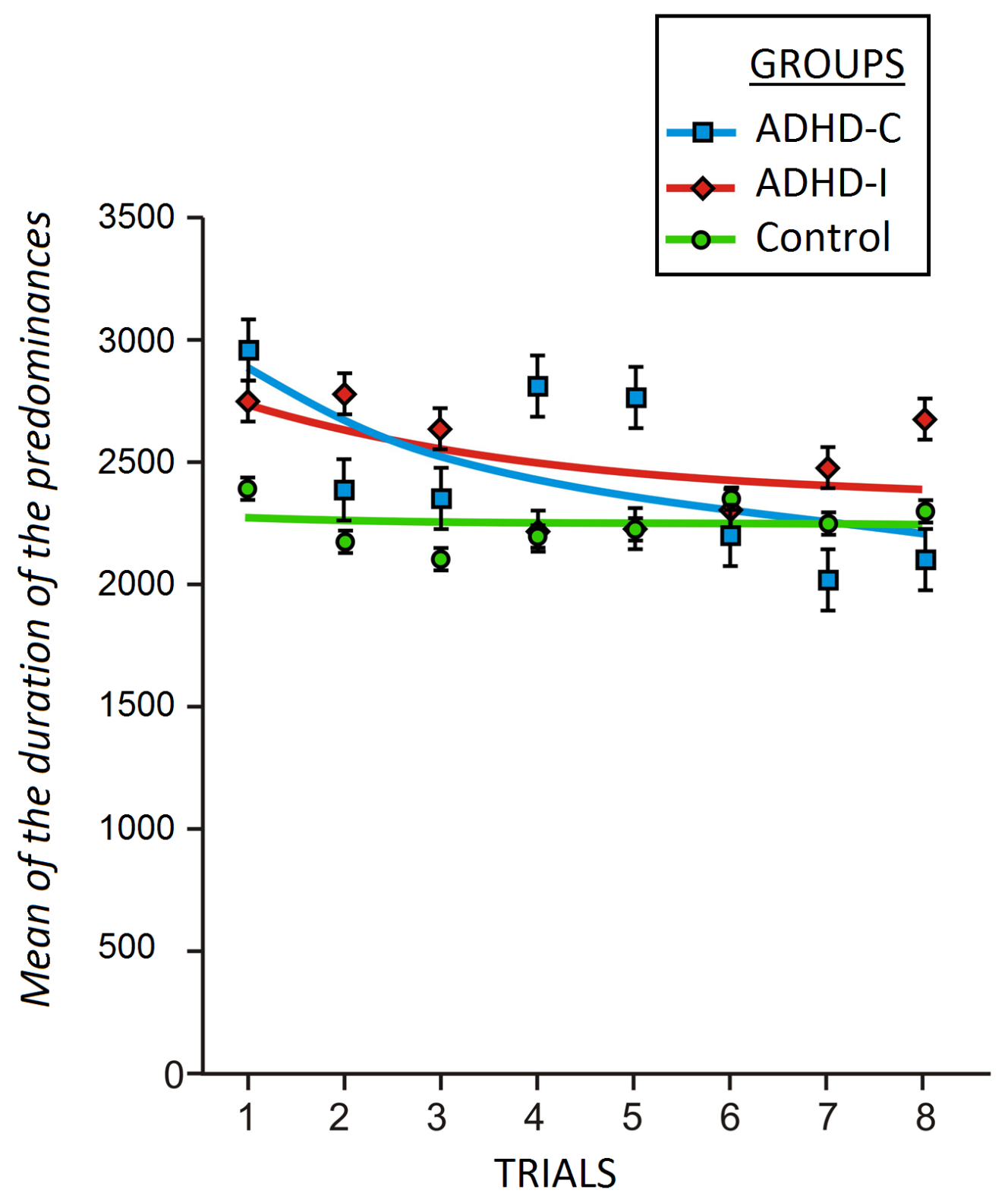

Figure 5. Effect of the 'group $x$ trial' interaction, based on the mean duration of exclusive dominance periods. The bars show the standard error. 